\title{
LHCb Vertex Detector Electronics Timing and Synchronization
}

\author{
Yuri Ermoline \\ Lausanne University
}

$8^{\text {th }}$ Pisa Meeting on Advanced Detectors

La Biodola, Isola d'Elba, Italy, May 21-27, 2000 


\section{LHCb Detector}

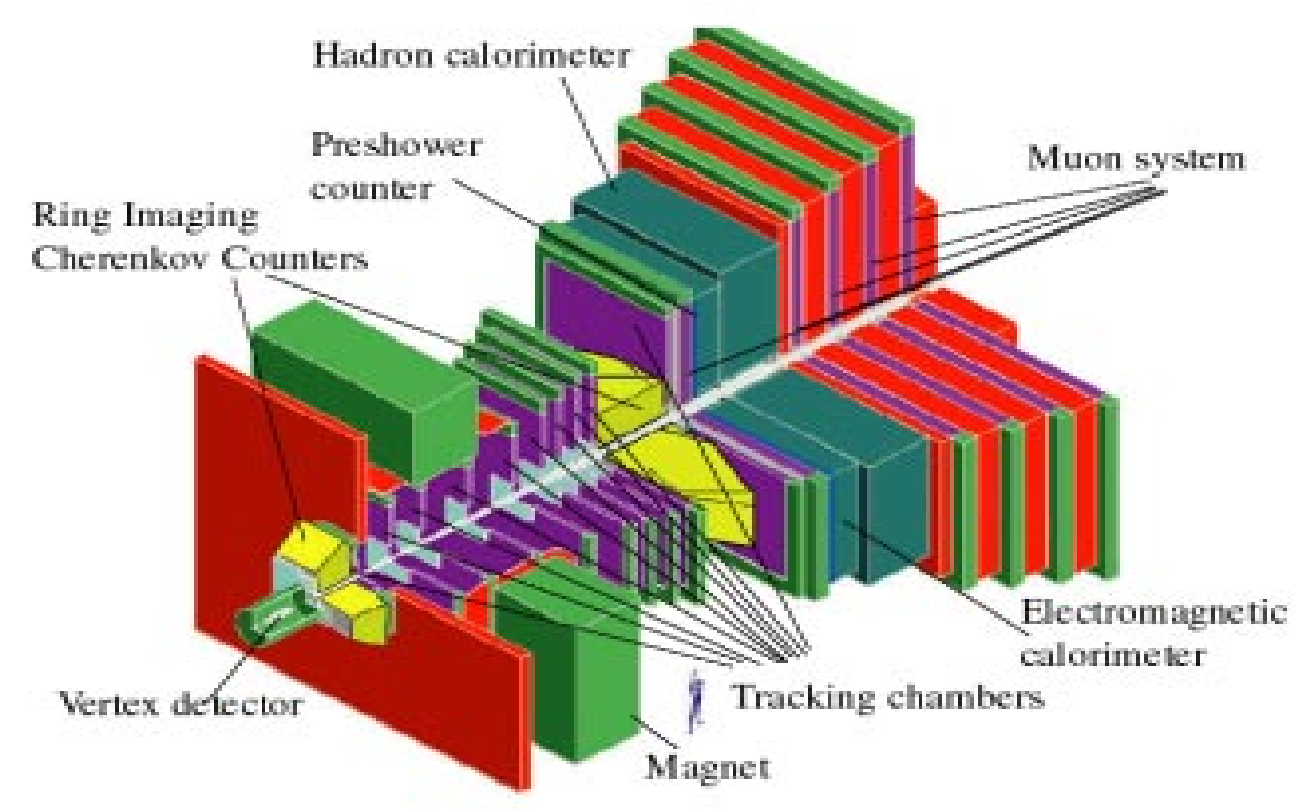

- CP Violation Experiment at the LHC

- Searches for rare B decays

a Single arm spectrometer

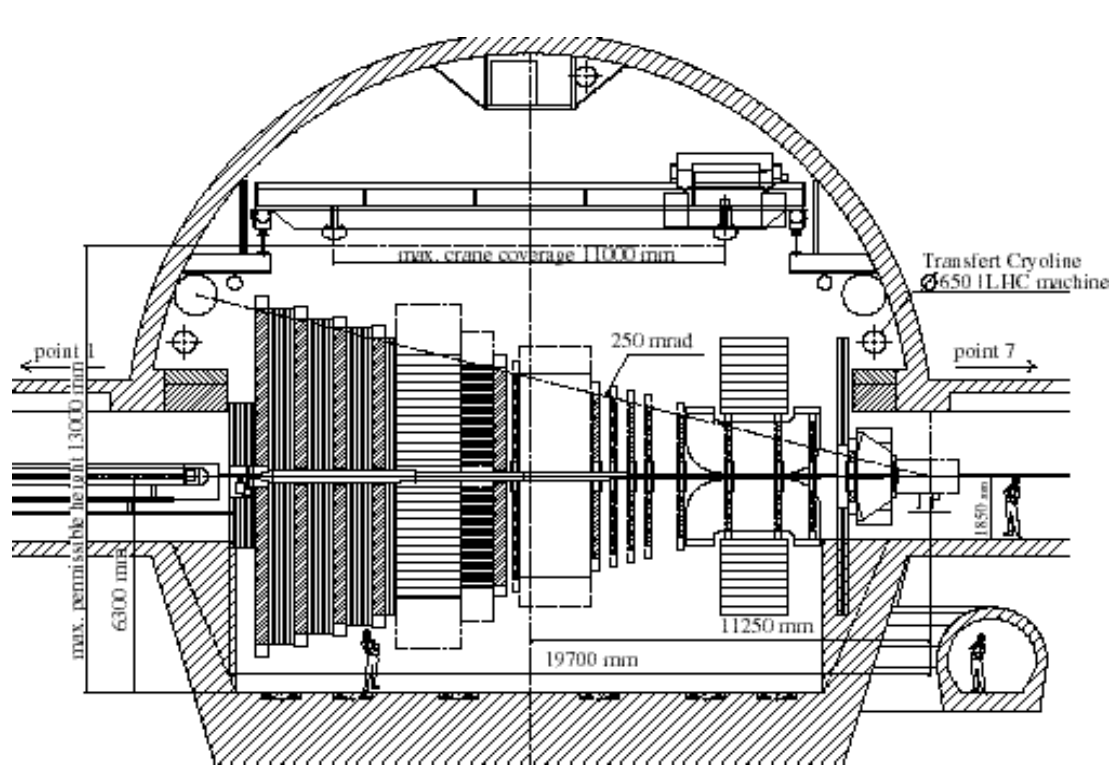

a Good decay time resolution

- Particle identification capability

a Good invariant mass resolution

a Flexible and optimized trigger 


\section{Trigger/DAQ Architecture}

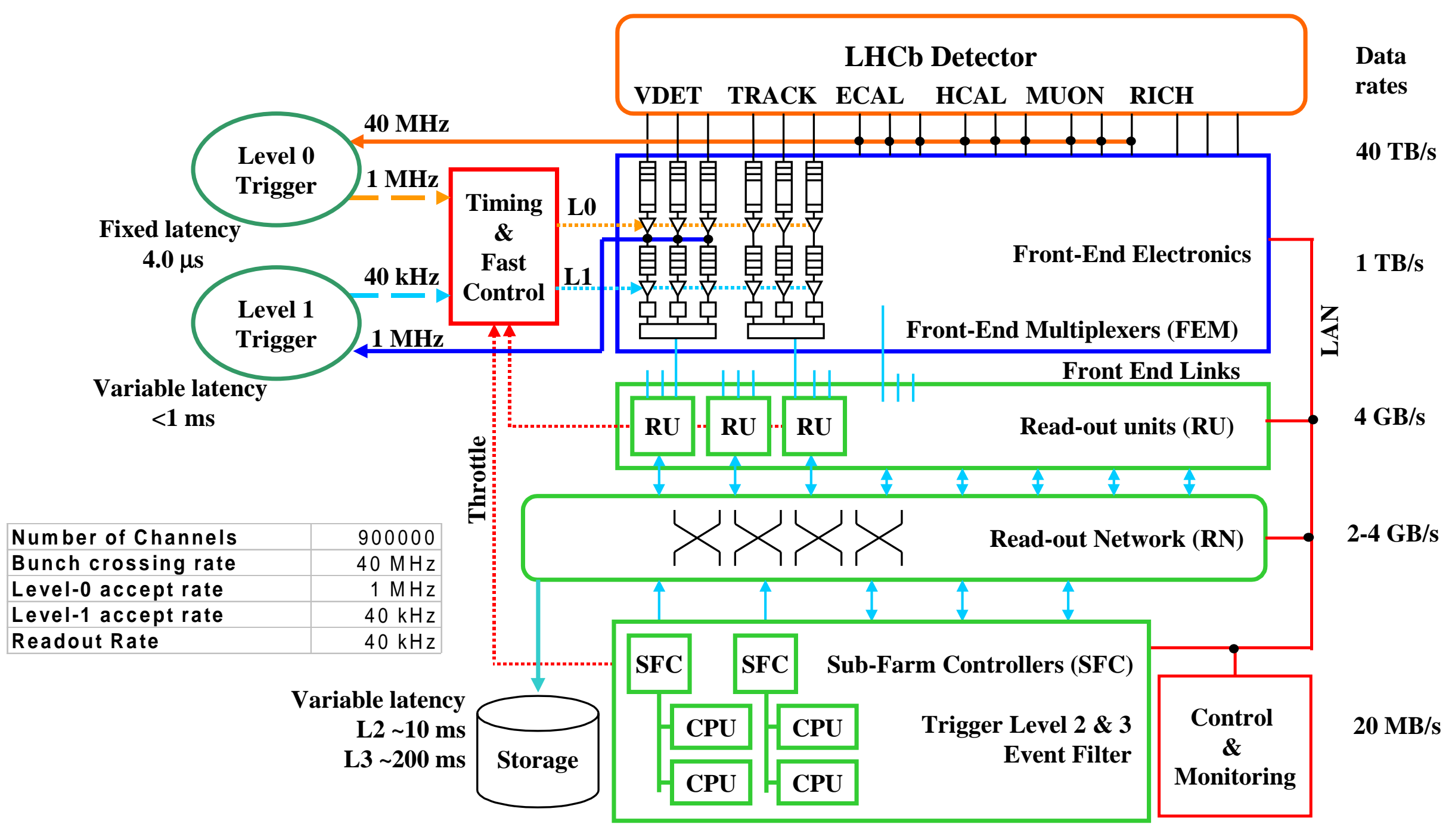




\section{Timing and Fast Control}

$\square$ Provide common and synchronous clock

$\square$ Provide Level-0 and Level-1 trigger decisions

- Provide commands synchronous in all components (Resets)

$\square$ Provide Trigger hold-off capabilities in case buffers are getting full

- TTC (CERN/RD-12) as a backbone

$\square$ LHCb specific Readout Supervisor and Readout Supervisor Switch

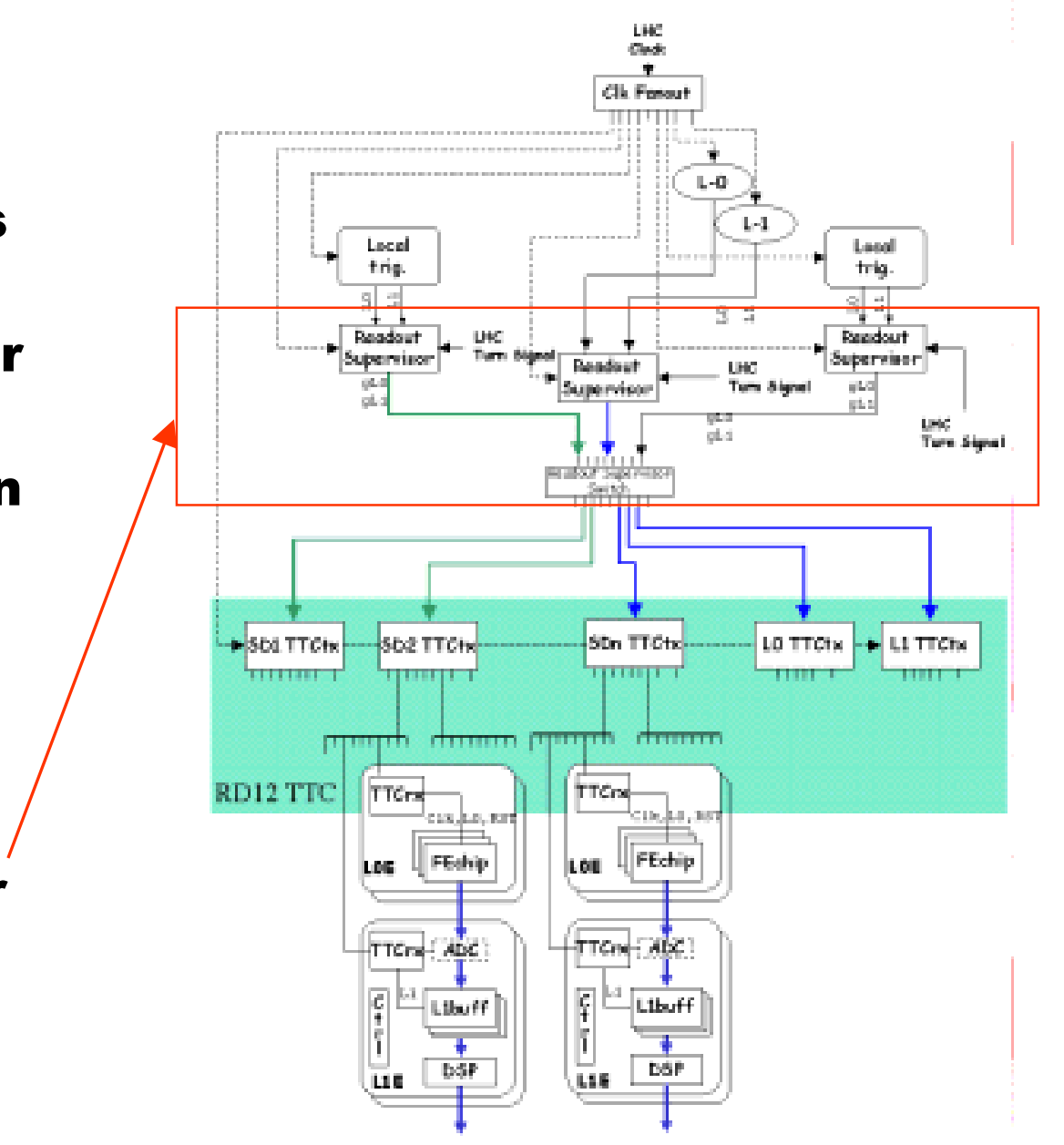




\section{VELO - VErtex LOcator}

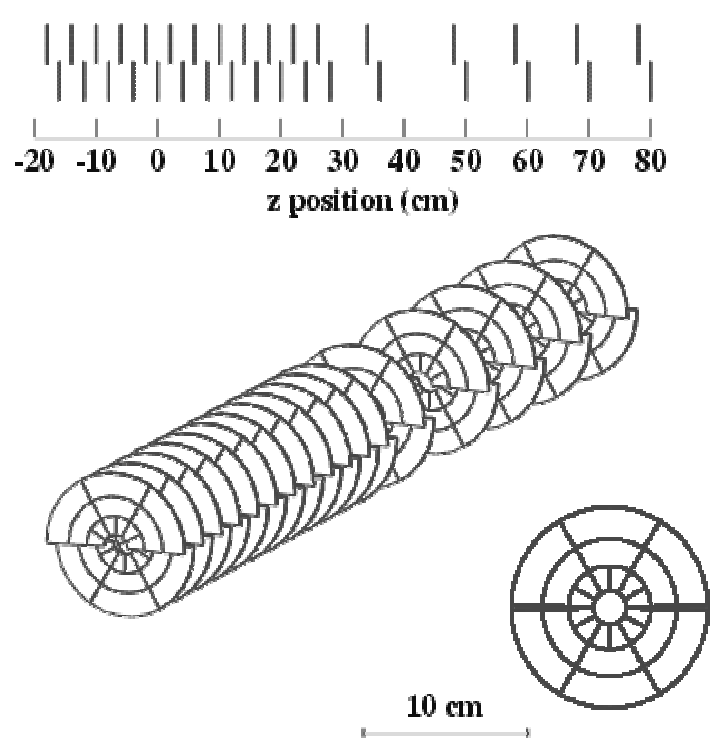

Typical event

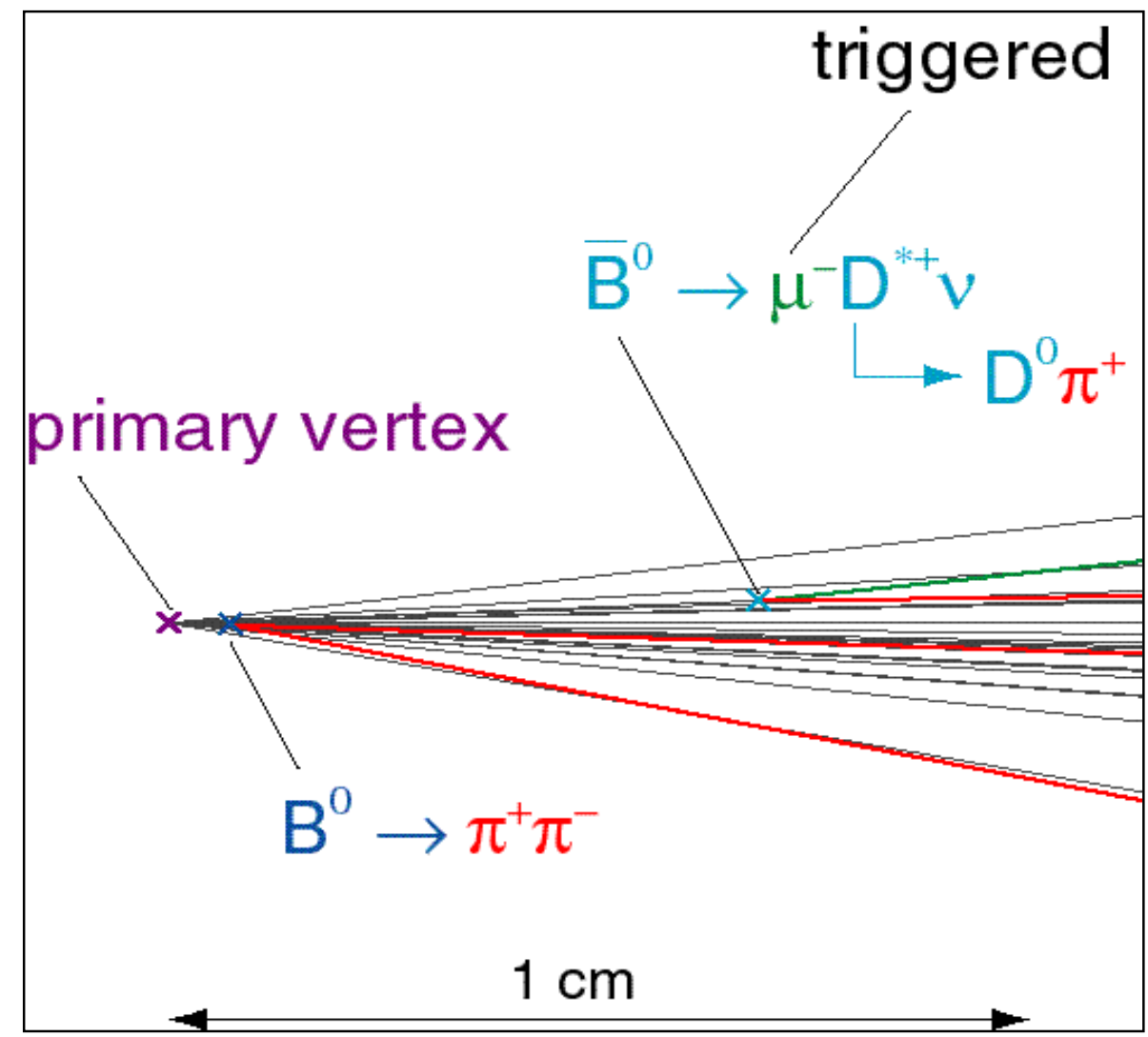




\section{VELO Electronics}

\section{Front-End Electronics}

O Radiation Hard (DMILL or deep sub-micron)

O 128 ch read-out chip with 4 outputs (32:1)

O 160 cells pipeline, 16 cells derandomizer

O Analogue read-out at $40 \mathrm{MHz}$

O Pipeline ID as Event ID, over 4 outputs

$900 \mathrm{~ns}$ read-out time $(32+4) * 25 \mathrm{~ns}$

O Clock, LOA, Reset from TTCrx

O Control via I2C interface

\section{- Read-Out (Off-Detector) Electronics}

O 64 channels module

8-bit 40MHz FADC (adj. Clock \& Vref)

O) Synchronisation tasks

L1T preprocessing

L1B for L1T latency (1000 events)

O Read-out \& transmission after L1A

a Processing Electronics

O DSP farm for zero-suppression

O Read-out electronics monitoring

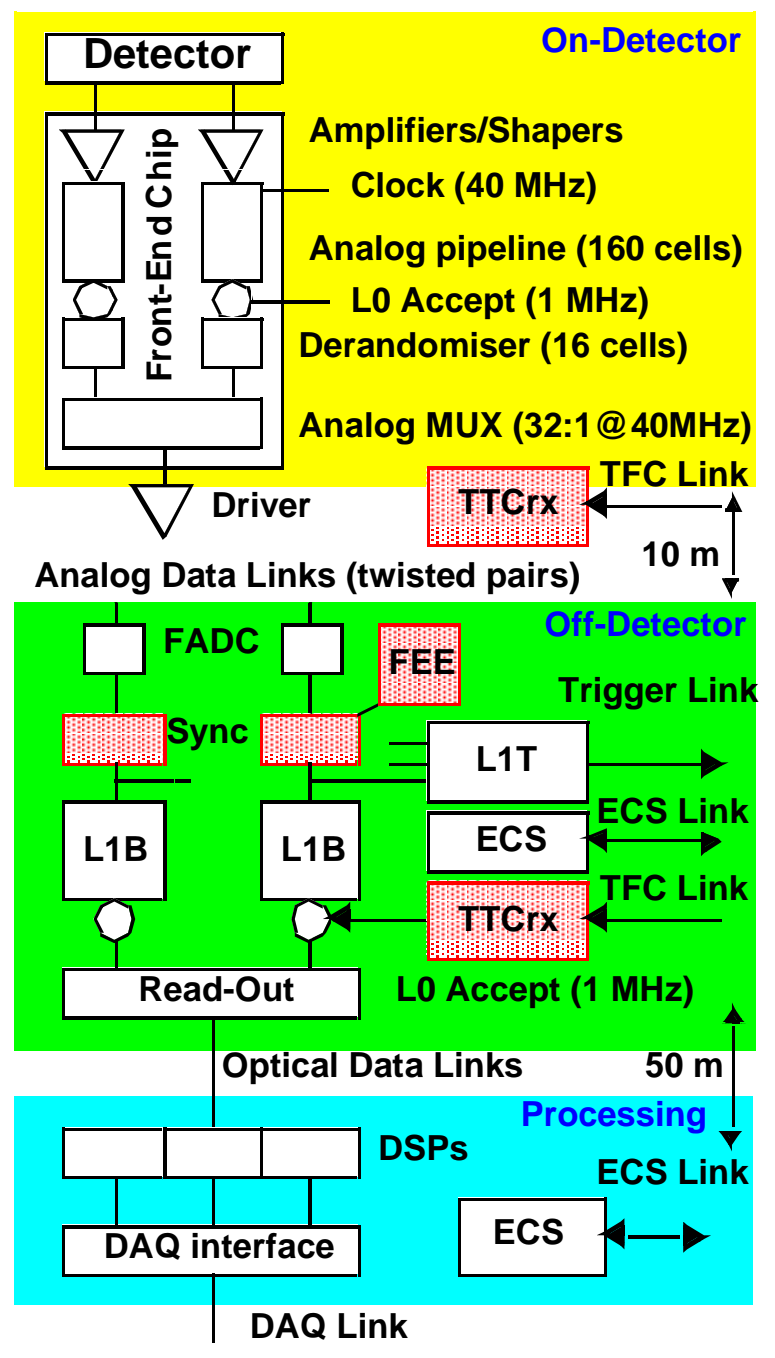




\section{Synchronization Tasks}

- Sampling synchronization

O Clock phase adjustment

O Set on signal model, adjuster on real

- LO Accept synchronization

o Lo Accept measurements

O LO Accept adjustment

a Front-end data synchronization

O FE Emulator time prediction

O FE data structure

aront-end error detection

O FE Event ID cross-check with ODE

- L1 Accept synchronization

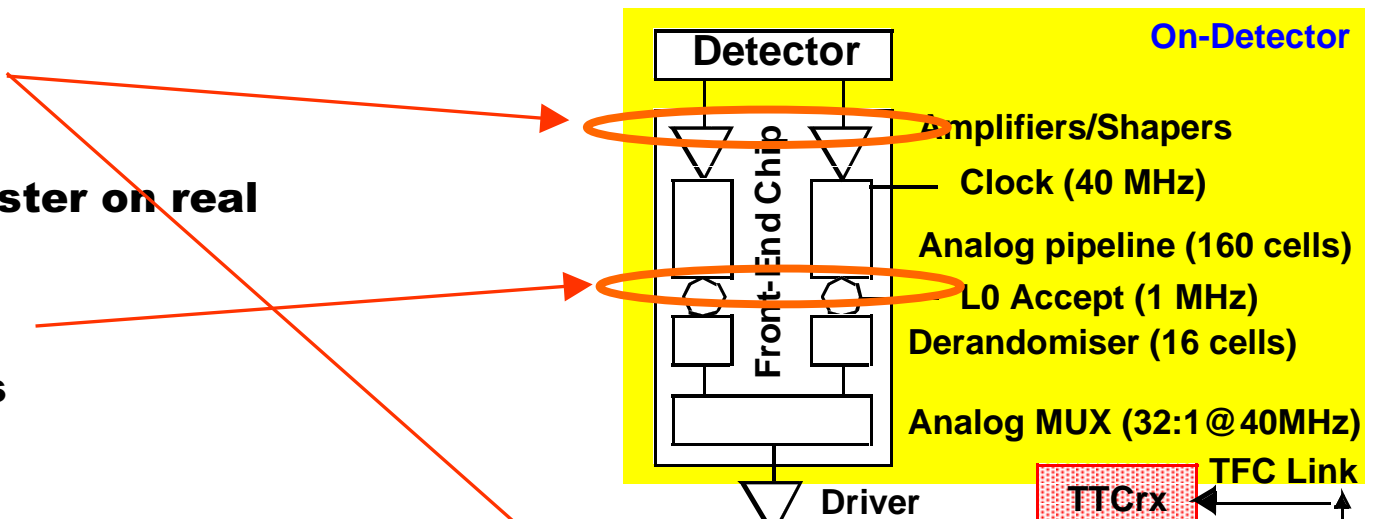

Analog Data Links (twisted pairs) $10 \mathrm{~m}$

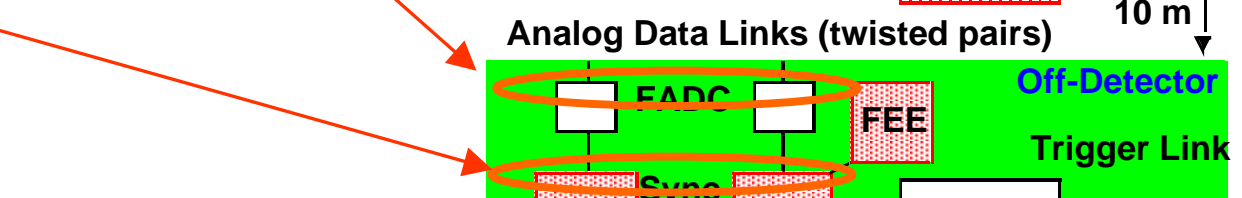

$\rightarrow$

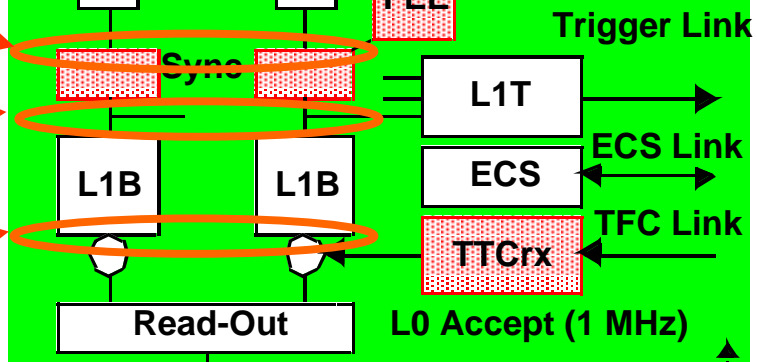

O Event ID + accept/reject

口 Event synchronization

Additional DAQ event number

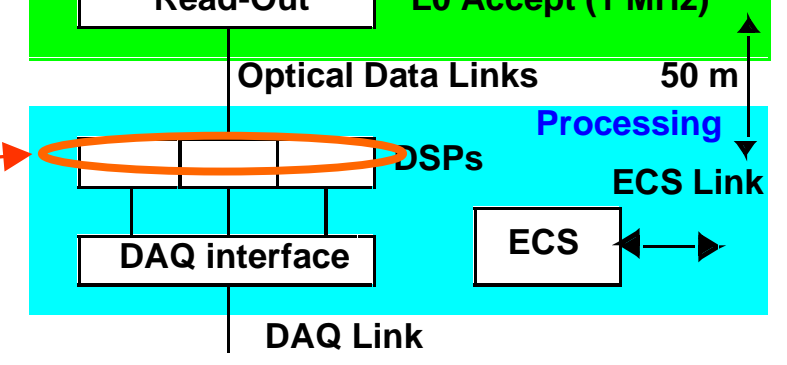




\section{Synchronization Hardware}

TTCrx receiver chip

O 0.1 ns clock adjustment

I Detector signal sampling

I FADC clock adjustment

O 16 clock cycles LO Accept delay

I LO Accept adjustment

O Bunch-crossing ID, LO and L1 Accepts

- Emulator of the FE chip control logic

- Predict FE data arrival time

I No need for separate strobe line

O Generate FE pipeline ID for cross-check

I FE chip error detection

O Generate Event ID

Synchronization logic

O FE chip synchronization errors detection

I Sets error flags for the L1 Buffer

I Masks the data for the L1 Trigger

Cvent tagging in the L1 Buffer

- L1 Accept synchronization

DAQ event number generation

On-Detector

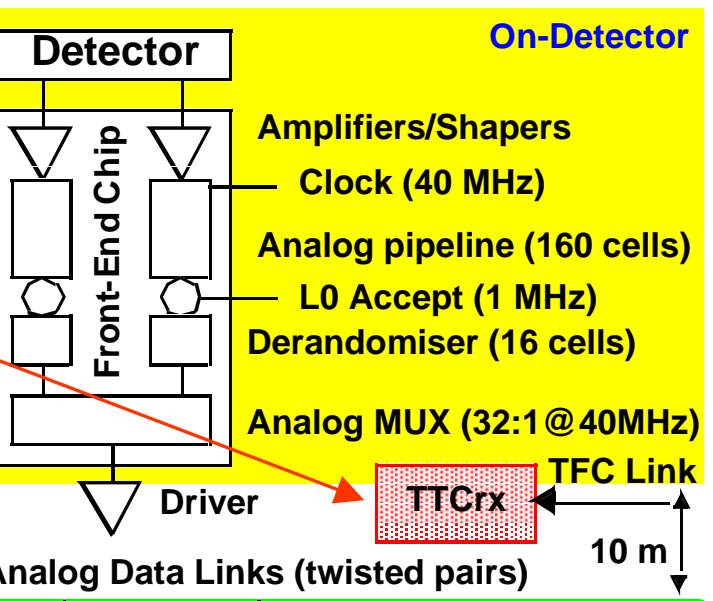

Off-Detector

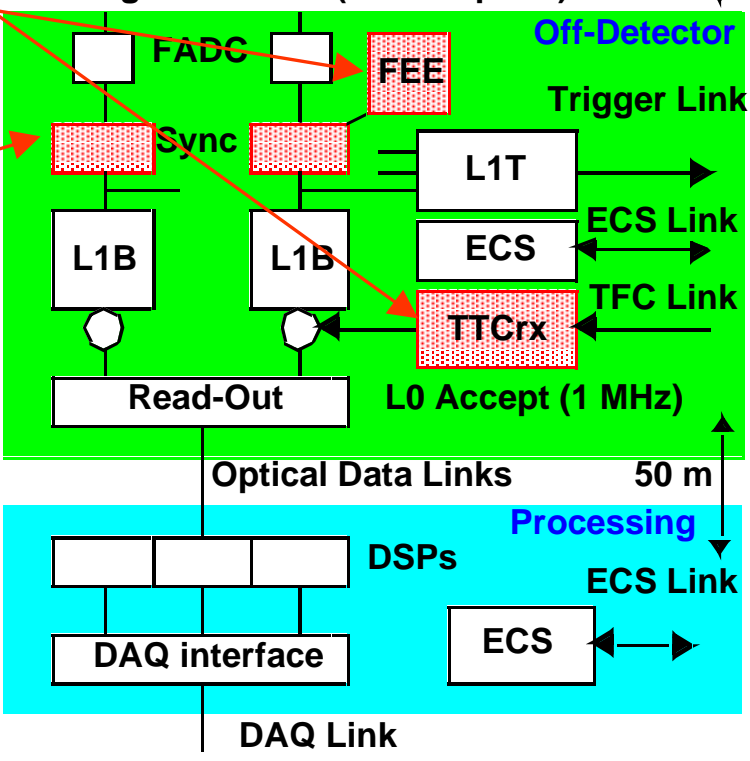




\section{Error Detection and Recovery}

a Synchronization errors are detected at different locations in the VELO

electronics

Corrupted data are flagged or masked

ㅁ Error statistics are collected via the Experiment Control System (ECS) and reported to the DAQ

The resetting frequency is calculated using observed error rate and different resetting scenarios are supported

- Resets are distributed to the VELO electronics using Timing and Fast Control system

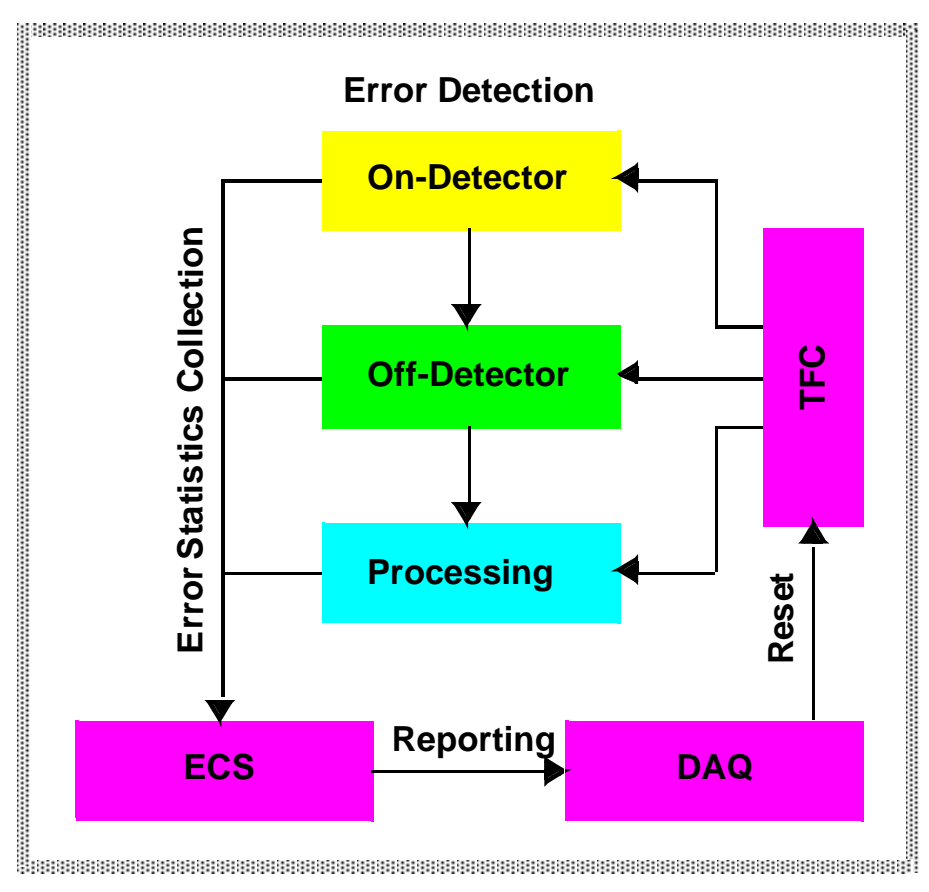




\section{Summary}

a VErtex LOcator (VELO) is a vital part of the LHCb experiment and it's Level-1 trigger

O Robustness is a major issue

VELO electronics located in a harsh radiation environment

Orrors are unavoidable

$\checkmark$ Synchronization check and error detection must be performed along the data flow

Special hardware are included in the data flow

Error recovery procedure involves periodical resets 